\section{Recent advances in clinical studies and the evolving role of subtyping for patients with diffuse large B-cell lymphoma}
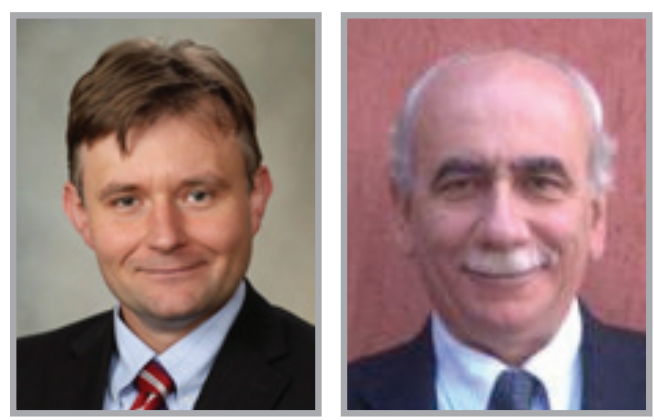

\author{
"It is critical that sick patients \\ enroll in present and future \\ clinical trials without being \\ excluded by aggressive \\ disease and perceived long \\ waiting times for molecular \\ marker evaluation."
}

Grzegorz S Nowakowski, \& Umberto Vitolo²

First draft submitted: 22 December 2016; Accepted for publication: 20 January 2017; Published online: 10 February 2017

The molecular classification of cell of origin (COO) for diffuse large B-cell lymphoma (DLBCL) has generated much enthusiasm for targeting new therapies to specific patient populations, such as those with activated $B$-cell $(A B C)$ versus germinal center B-cell (GCB) subtypes, double-expressors versus nonexpressors, and double-/ triple-hit lymphoma [1]. Indeed, the WHO revised classification of lymphoid neoplasms now requires identification of GCB and $A B C$ subtypes by immunohistochemistry (IHC) based algorithms (or alternately through gene-expression profiling [GEP] if accessible), and considers coexpression of $M Y C$ and BCL2 (i.e., double-expressor lymphoma) to be a new prognostic marker for DLBCL [2]. This classification also includes a new category for all double-/triple-hit high-grade B-cell lymphoma (other than follicular lymphoma or lymphoblastic lymphomas) with $M Y C$ and $B C L 2$ and/or BCL6 translocations.
However, results from several recent studies have dampened some of the excitement about the validity of these molecular markers and their role in clinical studies. So far, randomized studies have found no significantly improved survival outcomes with new chemoimmunotherapy regimens, including studies targeted to specific biological subtypes of DLBCL. Many studies were presented over the past 2 years at the American Society of Hematology annual meetings; final results of these studies are yet to be published.

After a median follow-up of 31.5 months, preliminary findings from the PYRAMID Phase II randomized controlled trial (RCT; NCT00931918) in patients with previously untreated non-GCB DLBCL uncovered no significant difference in the primary end point of progression-free survival (PFS) with R-CHOP versus bortezomib plus R-CHOP (VR-CHOP) at 2 years (77 vs 82\%; hazard ratio [HR]: 0.77 ; $90 \% \mathrm{CI}$ :

\section{KEYWORDS}

- chemotherapy

- hematologic/lymphoma

- molecular oncology

"The molecular classification of cell of origin for diffuse large B-cell lymphoma has generated much enthusiasm for targeting new therapies to specific patient populations, such as those with activated B-cell versus germinal center B-cell subtypes, double-expressors versus nonexpressors, and double-/triple-hit lymphoma” 
“Recent advances ... have made steps toward improving access for more aggressive types of disease to be enrolled in appropriate clinical trials, with the goal of making the trial more adherent to standard clinical practice and improving short- and long-term patient outcomes."
$0.45-1.30 ; p=0.70)[3]$. A lack of improvement with the addition of bortezomib was also shown in non-GCB patients who received VR-CAP versus R-CHOP [4]. Beyond the possibility that bortezomib might not be active in this population, study authors have speculated that the lack of effect might be caused by the impact of delays in IHC testing resulting in the selection of patients with less aggressive disease, possible limitations of IHC results as opposed to a gold standard GEP, both possibly resulting in a better than expected performance of the control R-CHOP arm. With a more reliable method of COO determination using GEP, a lengthy turnaround time in classifying patients can pose a significant treatment challenge. In the REMoDL-B study of R-CHOP \pm bortezomib, patients were stratified to $\mathrm{GCB}, \mathrm{ABC}$ or unclassifiable DLBCL subtypes by GEP while receiving their first cycle of R-CHOP therapy and before the start of cycle 2; median laboratory turnaround time for subtype classification was 12 working days [5]. While the approach used in the REMoDL-B study overcomes the issue of COO testing delay, as a first cycle of therapy was given regardless of DLBCL subtype, it is unknown how much use of bortezomib in first cycle of therapy is needed for its potential efficacy. Initial results from the REMoDL-B study showed no difference in response rates between the treatment arms. The study is maturing with regard to other end points. Final results from the GOYA RCT (NCT01287741) demonstrated no significant difference between obinutuzumab (G)-CHOP versus R-CHOP in the primary end point of investigator-assessed 3-year PFS (70 vs $67 \%$; HR: 0.92 ; $95 \%$ CI: $0.76-1.12$; $\mathrm{p}=0.3868)$ as first-line therapy in patients with DLBCL [6]. Interestingly, overall regardless of the type of treatment, COO assessed by GEP predicted different outcomes, with GCB patients having a better 3-year PFS: 75 versus 59\% in $\mathrm{ABC}$ and $63 \%$ in unclassified subtype (HR $\mathrm{ABC}$ vs GCB: 1.7 [95\% CI: 1.30-2.23]; unclassified versus GCB HR: 1.57 [95\% CI: 1.14-2.16]). A prespecified subgroup analysis of patients with GCB DLBCL (identified per GEP) showed a modest 3-year PFS improvement of $79 \%$ for G-CHOP versus $70 \%$ for R-CHOP (HR: 0.72; 95\% CI: 0.50-1.01).

Preliminary analysis from the CALGB/ Alliance 50303 RCT (NCT00118209) showed no difference in the primary end point of event-free survival (EFS) of R-CHOP versus dose-adjusted (DA)-EPOCH-R (HR: 1.02; $\mathrm{p}=0.89$ ) or the secondary end point of overall survival at a median follow-up of approximately 5 years [7]. The analysis by $\mathrm{COO}$ or $M Y C$ status was not reported at the time of the presentation.

So if we are failing to improve outcomes in first-line therapy of DLBCL, it is not for the lack of trying. While it appears that these novel combinations do not provide clinical benefit, careful analysis of the data helps to understand some of the limitations and potential pitfalls in designing future trials. In some cases, like in the PYRAMID and CALGB/Alliance 50303 trials, the control arm performed better than expected, either due to selection of a better than expected study population, limitations in the methods of determination of molecular subtype, or both (such as in the PYRAMID trial).

Is $\mathrm{COO}$ still valid method of identification for patients at high risk of relapse? A retrospective $\mathrm{COO}$ analysis of patient samples collected from two RCTs (RICOVER-60 and R-MegaCHOEP) by the German High Grade Lymphoma Study Group (DSHNHL) found significantly inferior survival for $\mathrm{ABC}$ over $\mathrm{GCB}$ DLCBL subtypes (identified via GEP using the Lymph2Cx Lymphoma Subtyping Test [LST] and the Nanostring nCounter ${ }^{\circledR}$ platform [NanoString Technologies, Inc.; Seattle, WA, US]) among CHOP-treated patients and no significant differences in EFS, PFS and overall survival by subtype among patients treated with R-CHOP or R-MegaCHOEP [8]. In contrast, a retrospective matched-cohort analysis of outcomes by COO (by LST Nanostring nCounter) showed worse EFS at 24 months for patients with $A B C$ versus GCB DLBCL (48 vs $71 \%$; $\mathrm{p}=0.013)$ who were treated with R-CHOP, but no difference between subtypes (69\% $A B C$ vs $67 \%$ GCB; $p=0.674)$ when patients received lenalidomide combined with $\mathrm{R}-\mathrm{CHOP}$ $\left(\mathrm{R}^{2}-\mathrm{CHOP}\right)$ [9]. Importantly, as mentioned above, prospective, prespecified analyses in the GOYA study, where $\mathrm{COO}$ was also assessed by LST Nanostring nCounter, showed prognostic value based on $\mathrm{COO}$ [6].

One lingering concern about RCTs in advanced DLBCL is whether or not they include the wider spectrum of patients found in everyday clinical practice. Findings from a recent examination of this point shed some light on this issue. An analysis of a large cohort of patients $(n=720)$ with stage II-IV DLBCL or primary mediastinal B-cell lymphoma treated 
with front-line anthracycline-based chemoimmunotherapy showed that patients who initiated therapy within 14 days of diagnosis had more aggressive disease characteristics compared with patients with delayed treatment initiation $(>14$ days from diagnosis) [10]. Less aggressive characteristics of patients with delayed treatment included earlier stage of disease, nonelevated lactate dehydrogenase, $\leq 1$ extranodal site, no B-symptoms, lower ECOG performance status, and lower International Prognostic Index (IPI), as well as an enriched GCB-specific population. Even after adjusting for IPI or age-adjusted IPI, patients with early treatment initiation had significantly worse EFS outcomes $(p<0.005)$. These results support the notion that RCTs that require a lengthy preinclusion work-up might be selecting for patients with less aggressive disease, which could generate a lower than expected event rate, thus undermining a study's statistical power.

How do we include high-risk, rapidly progressing patients in clinical trials? Considering very encouraging results previously reported with single arm (DA)-EPOCH-R (NCT00032019) [11], some centers hesitate to include high-risk patients into randomized trials with a R-CHOP control arm. However, results from the prospective, randomized CALGB/Alliance 50303 study showed no advantage to (DA)-EPOCH-R over R-CHOP [7]. Importantly, minimizing delays to initiation of therapy by introduction of rapid biomarker testing and pretreatment strategies aimed at stabilizing disease before chemotherapy can be initiated to provide an important tool for including high-risk, symptomatic patients in clinical trials. Some of the currently ongoing RCTs have been designed to minimize treatment delays to facilitate inclusion of patients with more aggressive disease, hopefully producing a more representative patient population. These studies are taking advantage of current molecular diagnostic techniques that can identify subtypes within days, thus minimizing any potential treatment delay based on molecular classification. For example, the ROBUST (NCT02285062) RCT of $\mathrm{R}^{2}$-CHOP21 versus $\mathrm{R}-\mathrm{CHOP} 21$ in patients with previously untreated ABC-type DLBCL incorporates a rapid central pathology review process for diagnosis, subtype, and CD20positive status completion within three calendar days of sample receipt [12]. To assist patients too sick to wait for therapy, the ROBUST protocol also allows investigators to use prephase corticosteroid treatment per local practice. Similarly, the ECOG E1412 Phase II trial of $\mathrm{R}^{2}$-CHOP versus R-CHOP in newly diagnosed DLBCL (NCT01856192) allows up to 7 days pretreatment with prednisone or equivalent for patients with systemic symptoms, compressive disease or rapidly progressing symptomatic adenopathy, without requiring a washout period.

In summary, while recently presented studies in DLBCL failed to improve outcomes, analysis of the results provides an important foundation for future studies. It is critical that sick patients enroll in present and future clinical trials without being excluded by aggressive disease and perceived long waiting times for molecular marker evaluation. Recent advances, such as allowance of pretrial steroids and more rapid turnaround of patient diagnostics, have made steps toward improving access for more aggressive types of disease to be enrolled in appropriate clinical trials, with the goal of making the trial more adherent to standard clinical practice and improving short- and long-term patient outcomes.

\section{Financial \& competing interests disclosure}

$G$ Nowakowski received research funding and travel expenses and has a consultingladvisory role at Celgene. $U$ Vitolo received research funding from Celgene and Roche, has had consulting or advisory roles at Roche and Janssen, and has participated in speaker's bureaus from Roche, Celgene, Janssen, Gilead and Takeda. The ROBUST study is supported by Celgene Corporation, Summit, NJ, USA. The authors have no other relevant affiliations or financial involvement with any organization or entity with a financial interest in or financial conflict with the subject matter or materials discussed in the manuscript apart from those disclosed.

Editorial support for this manuscript was provided by M Nicosia and J Kern, CMPP with Bio Connections LLC, which was funded by Celgene Corporation.

\section{References}

1 Nowakowski GS, Blum KA, Kahl BS et al. Beyond R-CHOP: a blueprint for diffuse large B cell lymphoma research. J. Natl Cancer Inst. 108(12), (2016).
2 Swerdlow SH, Campo E, Pileri SA et al. The 2016 revision of the World Health Organization classification of lymphoid neoplasms. Blood 127(20), 2375-2390 (2016).
3 Leonard JP, Kolibaba K, Reeves JA et al. Randomized Phase II open-label study of $\mathrm{R}-\mathrm{CHOP} \pm$ bortezomib in patients (Pts) with untreated non-germinal center B-Cell-like (non-GCB) subtype diffuse large cell 
lymphoma (DLBCL): results from the PYRAMID trial (NCT00931918). Blood 126(23), 811 (2015).

4 Offner F, Samoilova O, Osmanov E et al. Frontline rituximab, cyclophosphamide, doxorubicin, and prednisone with bortezomib (VR-CAP) or vincristine (R-CHOP) for non-GCB DLBCL. Blood 126(16), 1893-1901 (2015).

5 Davies AJ, Caddy J, Maishman T et al. A prospective randomised trial of targeted therapy for diffuse large B-cell lymphoma based on real-time gene expression profiling: the REMoDL-B study of the UK NCRI and SAKK lymphoma groups. Blood (ASH Annual Meeting Abstracts) 126(16), Abstract 812 (2015).

6 Vitolo U, TrnĚný M, Belada D et al. Obinutuzumab or rituximab plus $\mathrm{CHOP}$ in patients with previously untreated diffuse large B-cell lymphoma: final results from an open-label, randomized Phase III study (GOYA). Blood (ASH Annual Meeting Abstracts) 128(22), Abstract 470 (2016).

7 Wilson WH, Sin-Ho J, Pitcher BN et al. Phase III randomized study of R-CHOP versus DA-EPOCH-R and molecular analysis of untreated diffuse large B-cell lymphoma: CALGB/Alliance 50303. Blood (ASH Annual Meeting Abstracts) 128(22), Abstract 469 (2016).

8 Staiger AM, Ziepert M, Horn $\mathrm{H}$ et al. The clinical impact of the cell-of-origin classification and the MYC+/BCL2+ double expresser status in DLBCL treated within prospective clinical trials of the DSHNHL. Blood (ASH Annual Meeting Abstracts) 128(22), Abstract 151 (2016).

9 Nowakowski GS, Laplant BR, Pederson L et al. Lenalidomide combined with R-CHOP (R2CHOP) overcomes negative prognostic impact of $\mathrm{ABC}$ molecular subtype in newly diagnosed diffuse large B-cell lymphoma. Blood (ASH Annual Meeting Abstracts) 128(22), Abstract 3035 (2016).

10 Maurer MJ, Link BK, Habermann TM et al. Time from diagnosis to initiation of treatment of DLBCL and implication for potential selection bias in clinical trials. Blood (ASH Annual Meeting Abstracts) 128 (22), Abstract 3034 (2016).

11 Wilson WH, Jung SH, Porcu P et al. A CAncer and Leukemia Group B multi-center study of DA-EPOCH-rituximab in untreated diffuse large B-cell lymphoma with analysis of outcome by molecular subtype. Haematologica 97(5), 758-765 (2012).

12 Nowakowski GS, Chiappella A, Witzig TE et al. ROBUST: lenalidomide-R-CHOP versus placebo-R-CHOP in previously untreated ABC-type diffuse large B-cell lymphoma. Future Oncol. 12(13), 1553-1563 (2016). 